Sebastião Freitas de Medeiros ${ }^{1,2}$

Márcia Mariy Winck Yamamoto de MederRos 2

\title{
Modificações dos níveis de gonadotrofinas durante a vida reprodutiva
}

\author{
Gonadotropin level changes during the reproductive life
}

Palavras-chaves

Gonadorrelina

Receptores do LH Receptores do FSH Hormonio folículo estimulante humano Hormônio luteinizante

Keywords

Gonadorelin

Receptores, $\mathrm{LH}$

Receptores, FSH Follicle stimulating hormone Luteinizing hormone

\section{Resumo}

As modificações nas concentrações das gonadotrofinas ao longo da vida reprodutiva dependem de amadurecimento funcional harmônico entre conexões neurais, neurônios-GnRH no hipotálamo, gonadotropos hipofisários e células da granulosa e teca da parede folicular. As concentrações de LH e FSH sofrem variações com o período do dia, fase do ciclo menstrual e idades ginecológica e cronológica. A variação circadiana é marcante para o LH e ocorre na fase puberal, quando os pulsos têm maior freqüência e amplitude no período noturno. Na fase puberal tardia, os pulsos das gonadotrofinas ocorrem também durante o dia e perdem a variação noite-dia, permanecendo discretas alterações durante as 24 horas do dia. No ciclo menstrual, durante os anos reprodutivos, o FSH eleva-se no final da fase lútea tardia, declina na fase folicular média, eleva-se bruscamente na fase ovulatória e permanece em níveis basais até a fase lútea tardia. $\bigcirc \mathrm{LH}$ permanece em níveis constantes durante toda a fase folicular, eleva-se no pico ovulatório e declina a níveis basais na fase lútea. Na quarta década, há modificações hipotálamo-neurais na secreção de GnRH, atenuação do retrocontrole positivo exercido pelo estradiol e diminuição na freqüência e prolongamento dos pulsos de GnRH. A hipófise responde com diminuição na densidade de receptores-GnRH, perda da sensibilidade do gonadotropo, secreção de gonadotrofinas mais básicas com maior meia-vida, diminuição na freqüência e maior amplitude dos pulsos de LH e FSH e secreção preferencial de FSH. Estas modificações, associadas à aceleração no consumo folicular, explicam a elevação monotrópica mais rápida de FSH após os 37-38 anos e a manutenção de níveis quase constantes de $\mathrm{LH}$ até o final do período reprodutivo. Estudos consistentes mostram que a elevação seletiva dos níveis basais de FSH na fase folicular precoce na verdade é gradual, sendo observada já na terceira década de vida. A discordância entre pulsos e níveis basais de FSH e LH ao longo dos anos reprodutivos permite especular a existência de mecanismos mais complexos, não totalmente compreendidos, na regulação do eixo hipotálamo-hipófise-ovariano.

\section{Abstract}

Changes in the levels of gonadotropins throughout the reproductive life depend on a fine tuned functional development of neural pathways and $\mathrm{GnRH}$-neurones, pituitary gonadotrophs and granulosa-theca cells of the follicular wall. Both, LH and FSH levels change according to the day-time, menstrual cycle phase, and gynecological age. Initiating the puberty, changes in LH pulses are remarkable, showing higher frequency and amplitude at night. Later in puberty, the pulses of $L \mathrm{H}$ are also maintained during the day, remaining its levels with very little variation within the 24 hours period. During the menstrual cycle, the FSH levels increase at the end of the luteal phase, decrease during the medium and late follicular phase, increase rapidly in the ovulatory phase and remain at low basal levels until the late luteal phase. The levels of $\mathrm{LH}$ remain unaltered during the whole follicular phase, increase in the ovulatory surge, and decrease to the basal levels in the luteal phase. At the forth decade of life, the GnRH secretion changes, with hypothalamic loss of sensitivy to the estradiol positive feedback and decrease in frequency and prolongation of the $\mathrm{GnRH}$ pulses. The pituitary response is atenuated due to decrease in the density of $\mathrm{GnRH}$ receptors on gonadotroph cells, loss of gonadotroph sensitivity, secretion of more basic FSH and LH molecules, decrease in frequency and increase in amplitude of $\mathrm{LH}$ and FSH pulses. These modifications result in monotropic increase of the FSH secretion. Current studies show that the selective increase in the FSH levels in the early follicular phase is gradual, beginning as early as the third decade of life. These alterations in FSH are associated with an accelerated follicular depletion in women after 37-38 years old. On the other side, the $\mathrm{LH}$ levels remain almost constant up to the end of reproductive life. The different levels of FSH and $\mathrm{LH}$ seen throughout the reproductive years may be due to yet unknown regulatory mechanisms in the hypothalamic-pituitary-ovarian axis.

Correspondêncio: Sebastiōo Freitas de Medeiros Rua Marechal Deodoro, 1055, apto. 1302 - Centro CEP 78005-101 - Cuiabá/M Fone: (65) 3322-6043 - Fax: (65) 3623-0079 E-mail: de.medeiro@@terra.com.br
Professor Adjunto, Departamento de Ginecologia e Obstetrícia, Faculdade de Ciências Médicas, UFMT. Diretor do Instituto Trapical de Medicina Reprodutiva e Menopausa, Cuiabá (MT), Brasil.

Diretora do Laboratório de Reprodução Assístida do Instituto Tropical de Medicina Reprodutiva e Menopausa, Cuiabá (MT), Brasil. 


\section{Introdução}

Biomarcadores biológicos para as modificações endócrinas ao longo da vida são pouco compreensíveis devido à heterogenidade populacional e à influência dos fatores ambientais nas populações estudadas. As funções do eixo hipotálamo-hipófise-ovariano (HHO) envolvem neurônios do hipotálamo médio basal, gonadotropos hipofisários e células teca-granulosas de um número variável de folículos ovarianos de acordo com o período da vida e a fase do ciclo menstrual. No sistema endócrino-reprodutivo, as gonadotrofinas sofrem variações qualitativas e quantitativas específicas segundo o período de vida, obedecendo a modulação do hipotálamo, da própria hipófise e dos esteróides ovarianos. As modificações percebidas ao longo dos anos na função do eixo $\mathrm{HHO}$ envolvem tanto alterações na sensibilidade do sistema de retrocontrole como mudanças nos tecidos alvo ou metabolismo/degradação dos hormônios envolvidos ${ }^{1}$. Um declínio na função reprodutiva nas mulheres torna-se evidente cerca de dez anos antes do seu término. As modificações endócrinas repercutem diretamente em manifestações clínicas. De fato, a fertilidade nas mulheres diminui durante a quarta década de vida, período associado com importantes modificações na secreção de gonadotrofinas ${ }^{2}$. A velocidade de consumo folicular ovariano, variável nos diferentes períodos de vida, é maior na segunda metade da quarta década, estando associada à elevação do hormônio folículo estimulante (FSH), não acompanhada de qualquer modificação do hormônio luteinizante $(\mathrm{LH})^{3}$. Esta aceleração no consumo folicular parece ser conseqüência de modificações no hipotálamo e no sistema nervoso central (SNC) relacionadas à idade.

Estudos experimentais em animais, tanto transplantando ovários de ratas idosas com ciclos anormais para ratas jovens castradas, como pela administração de drogas restaurando a atividade monoaminérgica hipotalâmica ou a estimulação elétrica da área hipotalâmica pré-ótica em animais idosos, demonstraram ser possível reiniciar ciclos estrais mensais ovulatórios ${ }^{4}$. Em mulheres, modificações nas gonadotrofinas antes de qualquer modificação no padrão menstrual têm sido também relatadas e atribuídas a alterações hipotalâmicas e/ou hipofisárias devidas também à idade ${ }^{5}$. Diante do acúmulo de evidências acerca da deterioração funcional do eixo hipotálamo-hipofisário feminino com o passar dos anos, repercutindo na dinâmica das gonadotrofinas ao longo da vida, esta revisão tem como objetivos:

1 examinar os mecanismos fisiológicos do eixo $\mathrm{HHO}$;

2 analisar, por compartimento, as repercussões da idade na secreção do hormônio liberador de gonadotrofinas $(\mathrm{GnRH})$, gonadotrofinas e esteróides sexuais;

3 caracterizar as modificações nos níveis das gonadotrofinas durante a vida reprodutiva;

4 apontar os conteúdos nos quais novos estudos são ainda necessários.

\section{Função gonadotrópica hipotálamo-hipofisária}

Após a formação do hipotálamo, cerca de 800-3000 neurônios capazes de sintetizar GnRH estão distribuídos na área pré-ótica e/ou hipotálamo médio basal, constituindo, no conjunto, o pulso gerador de GnRH. Estes neurônios são conectados entre si ou a outros neurônios dentro e fora do hipotálamo e expressam receptores mobilizadores de cálcio (catecolaminas, $\alpha$-1adrenérgicos e endotelina), ativadores do sistema adenilciclase ( $\beta$-adrenérgico, dopaminérgico), da tirosinoquinase (insulina, fatores de crescimento insulina-símiles I e II, fator de crescimento epidérmico, fator de crescimento dos fibroblastos e prolactina $)^{6}$. Receptores para os aminoácidos aspartato e ácidos gama amino butírico A e B (GABA) e esteróides sexuais são também expressos neste conjunto neuronal. Todos estes elementos exercem funções autócrinas e parácrinas na secreção de $\mathrm{GnRH}^{7}$. Em adição, a secreção pulsátil, intrínseca do $\mathrm{GnRH}$, está também associada à existência de atividade elétrica episódica espontânea no hipotálamo ${ }^{8}$.

$\mathrm{Na}$ área hipofisiotrópica hipotalâmica, há processamento das informações percebidas do meio interno do indivíduo e ambiente externo (hora do dia, duração do dia, estação do ano e impacto de emoções) via neurotransmissores e esteróides sexuais. Mais precisamente, estas variáveis modulam os neurônios do núcleo arqueado no hipotálamo médio-basal, resultando na síntese e secreção pulsátil de GnRH, em ritmo de um pulso a cada 60-90 minutos 9 . Em síntese, os sistemas neuronais do tronco cerebral mais complexos adrenérgicos e dopaminérgicos são os principais moduladores da função pulsátil dos neurônios-GnRH ${ }^{10}$. O sistema neural adrenérgico do tronco cerebral projeta-se diretamente aos neurônios $\mathrm{GnRH}$ e GABA, sendo que a noradrenalina tanto estimula o sistema $\mathrm{GnRH}$ quando atua diretamente, como inibe-o quando atua via neurônios GABA. A dopamina, neurotransmissor dopaminérgico, inibe a síntese e/ou liberação do $\mathrm{GnRH}$ na eminência média e circulação porta-hipofisária. O sistema opiodérgico, cujos neurônios estão localizados nos núcleos supraótico e para ventricular, inibe o sistema $\mathrm{GnRH}$.

As ações dos esteróides sexuais nos neurônios-GnRH são intermediadas principalmente pelos neurônios GABA. Assim, a maioria dos impulsos originados fora da área pré-ótica sobre os neurônios-GnRH são canalizados por via destes neurônios. A maioria dos 
neurônios GABA da área pré-óptica expressam receptores para os esteróides sexuais e sofrem estímulos destes hormônios, explicando, parcialmente, o efeito de retrocontrole negativo destes sobre a secreção de GnRH. A identificação recente de receptores esteróides nos próprios neurônios-GnRH sugere possível ação direta. $\mathrm{Na}$ adenohipófise, o gonadotropo sofre modulação do GnRH via circulação porta-hipofisária. Nesta célula, a molécula de GnRH liga-se a receptores específicos na membrana, ativa o sistema proteína-G e mobilização de cálcio e proteína cinase C, induzindo a expressão de mRNA para a síntese das subunidades alfa-glicoproteínas, $\beta \mathrm{LH}$ e $\beta \mathrm{FSH}^{11}$. A interação GnRH-receptor no gonadotropo altera o número dos receptores disponíveis e a própria sensibilidade do gonadotropo ao GnRH. Enquanto a exposição contínua do GnRH dessensibiliza o gonadotropo, a exposição pulsátil previne a dessensibilização e aumenta a sensibilidade 9 . Pulsos mais freqüentes de GnRH favorecem a secreção de LH e pulsos mais esparsos favorecem a secreção de FSH. Na prática, a pulsatilidade na secreção de FSH é mais difícil de ser documentada devido à sua maior meia vida. Flutuações cíclicas na amplitude e freqüência dos pulsos de GnRH, combinadas com mudanças locais na capacidade secretora do gonadotropo e níveis variáveis de esteróides sexuais respondem pelo perfil de secreção das gonadotrofinas ${ }^{10}$.

Os esteróides gonadais alcançam a hipófise e também modulam a resposta do gonadotropo ao GnRH. $\mathrm{O}$ estradiol estimula a síntese e o armazenamento das gonadotrofinas, mas impede a liberação destas glicoproteínas na circulação sistêmica, promovendo, então, o acúmulo destas moléculas no interior das células. A progesterona, caso o gonadotropo tenha sido previamente exposto ao estradiol, e quando ainda em pequenas quantidades, favorece a síntese de FSH. Em níveis maiores que $0,8-1,0 \mathrm{ng} / \mathrm{mL}$ inibe a atividade do gonadotropo e diminui a freqüência dos pulsos de $\mathrm{LH}$ e $\mathrm{FSH}^{12}$. Os androgênios, de modo geral, inibem a síntese de gonadotrofinas. Como a secreção de FSH e LH pelo gonadotropo obedece aos pulsos de $\mathrm{GnRH}$ modulados pelos esteróides sexuais, a concentração das gonadotrofinas sofre variação segundo a fase do ciclo menstrual. Além da influência dos esteróides sexuais, a secreção de FSH pelo gonadotropo, mas não a de LH, sofre modulação dos peptídeos ovarianos ativina, inibina e folistatina ${ }^{13}$.

A função ovariana basal é contínua em todos os momentos de sua existência. Assim, os ovários têm sua própria atividade e regulação local. Nos anos reprodutivos, a resposta ovariana aos pulsos de $\mathrm{LH}$ e
FSH ocorre de maneira variável em ciclos de aproximadamente 28 dias e esta ciclicidade sobrepõe-se à atividade basal gonadotrofino-independente. Logo, hipotálamo e hipófise, via modificações nos níveis de LH e FSH, controlam e modulam apenas a etapa final do crescimento folicular, a ovulação e a fase lútea. $\mathrm{O}$ FSH é o hormônio chave para o crescimento folicular, sendo que seus níveis basais refletem o estoque folicular de ambos os ovários em um determinado momento da vida reprodutiva. À medida que diminui a reserva folicular, os níveis basais de FSH se elevam. Os níveis basais de LH, relevantes na fase folicular tardia, permanecem mais estáveis e elevam-se tardiamente na vida reprodutiva, já no período pré-menopausa ${ }^{2,3}$.

Repercussões do envelhecimento na função hipotálamo-hipófise-ovariana

O processo de senescência do sistema reprodutor, envolvendo o SNC, hipotálamo, hipófise e ovários, é caracterizado clínica e bioquimicamente por alteração na ciclicidade ovulatória, modificações no intervalo do ciclo menstrual, diminuição da sensibilidade dos folículos ovarianos ao FSH, elevação seletiva de FSH desconectada da elevação de LH e diminuição ou perda da capacidade reprodutora. No hipotálamo, alterações no sistema secretor de $\mathrm{GnRH}$ têm início mesmo antes dos primeiros sinais da falência ovariana. Há modificações tanto na biossíntese, transporte e liberação de $\mathrm{GnRH}$, quanto nos fatores reguladores adrenérgicos, dopaminérgico ou na influência dos fatores de crescimento ${ }^{14}$. A idade pode afetar ainda a expressão dos receptores estrogênicos ou de seus coativadores? ${ }^{9}$. Algumas modificações, idade dependentes, a exemplo do que acontece no sistema neuronal opioidérgico, ocorrem precocemente, ainda que o número de neurônios permaneça constante. No entanto, o número de neurônios noradrenérgicos, localizados no locus coeruleus, pode diminuir com a idade, influenciando a secreção de $\mathrm{GnRH}^{15}$.

Disfunções hipotalâmicas com o envelhecimento reprodutivo ocorrem de modo independente do consumo folicular ${ }^{16}$, sendo estas disfunções marcapasso de uma cascata de eventos que resultarão na falência reprodutiva. Peptídeos neurotransmissores expressos ou conectados ao núcleo supraquiasmático, responsável pela ritmicidade dos pulsos hipotalâmicos, projetam-se para os neurônios-GnRH. A deterioração deste sistema, que assegura a função rítmica, leva à diminuição da capacidade reprodutora e alterações do ciclo menstrual. Em ratas adultas idosas, a resposta do sistema GnRH durante o pico de estradiol é significativamente menor; do mesmo modo, os pulsos de GnRH estão diminuídos nestes animais quando comparados com os pulsos 
observados em ratas mais jovens ${ }^{17}$. Logo, o sistema de retrocontrole positivo estrogênico é atenuado com o envelhecimento. A atividade elétrica intrínseca do sistema gerador dos pulsos de GnRH está também diminuída nas ratas idosas ${ }^{18}$.

Em humanos e primatas não-humanos, o papel do hipotálamo na senescência reprodutora é menos claro. Em macacas Rhesus há elevação na freqüência pulsátil do GnRH tardiamente, já na perimenopausa. Em mulheres, ainda na quarta década da vida, há diminuição na freqüência e prolongamento da ação dos pulsos de $\mathrm{GnRH}$, refletindo deterioração na coordenação entre os neurônios-GnRH e seus moduladores centrais ${ }^{19}$, com atenuação da atividade do pulso gerador de $\mathrm{GnRH}^{20}$. O resultado desta modificação funcional é a elevação seletiva de FSH quando mais de 25.000 folículos estão ainda presentes nos ovários 5 . Com o passar do tempo, há também, na mulher, perda da sensibilidade dos neurônios-GnRH aos esteróides sexuais, tornando atenuada ou ausente a resposta de retrocontrole positivo hipotalâmico ao pico de estrogênio ${ }^{19}$, possivelmente por alteração no sistema catecolaminérgico ${ }^{16}$, diminuição sináptica entre os neurônios adrenérgicos e neurônios-GnRH e alteração do sistema opioidérgico ${ }^{15}$. Uma demonstração do envelhecimento funcional hipotalâmico é o fato de que as modificações descritas são menos robustas nas mulheres com menopausa precoce ${ }^{21}$. Além disso, há perda parcial da capacidade de supressão da elevação das gonadotrofinas com a administração exógena de estradiol nas mulheres mais idosas.

Como visto, a hipófise integra e processa vários sinais neuroendócrinos via esteróides ou peptídeos com funções autócrina, parácrina e endócrina. A função do próprio gonadotropo sofre modificações com a idade, refletindo negativamente na secreção de gonadotrofinas e regulação da função ovariana ${ }^{11}$. Há diminuição do número de receptores para o $\mathrm{GnRH}$, perda da sensibilidade do gonadotropo ao $\mathrm{GnRH}$ e modificações qualitativas nas moléculas de FSH e LH. A diminuição dos receptores para o GnRH no gonadotropo favorece a síntese das moléculas de $\mathrm{FSH}^{1,22}$. A meia vida do $\mathrm{LH}$ parece prolongada nos últimos anos da fase reprodutiva ${ }^{20}$. Além das modificações nas gonadotrofinas e diminuição da resposta ao $\mathrm{GnRH}$, a senescência hipofisária reflete diretamente na diminuição da fertilidade.

Considerando que as duas gonadotrofinas podem ser sintetizadas pelo mesmo gonadotropo, a elevação do FSH não acompanhada da elevação do LH pode ser atribuída à existência de controle distinto, diferenças na pulsatilidade e/ou amplitude de $\mathrm{GnRH}$, diferença na densidade dos receptores no gonadotropo ou a diferenças no metabolismo das gonadotrofinas ${ }^{1,23}$. Na perimeno- pausa, há diminuição na freqüência dos pulsos de LH e prolongamento, por maior amplitude, dos pulsos de $\mathrm{FSH}^{20}$. Na pós-menopausa, há elevação da responsividade ao GnRH e aumento na taxa de produção e clearance renal devido à secreção de moléculas mais básicas, que têm maior sobrevida ${ }^{24}$. Mulheres com menopausa após os 40 anos secretam menor quantidade e pulsos de LH e FSH com menor freqüência do que aquelas com menopausa precoce. Em adição, a secreção de $\beta \mathrm{LH}$ e $\beta \mathrm{FSH}$ e alfa subunidades livres declina progressivamente após a menopausa ${ }^{25}$.

Na primeira metade da gravidez, há formação de folículos ovarianos a partir da interação entre as células germinativas que alcançam a crista gonadal e as células somáticas. Pouco antes da $20^{a}$ semana de gestação encerra-se a formação de novos folículos, tendo início consumo gradual da população previamente formada. A velocidade de consumo folicular, modulada por mecanismos endócrinos, parácrinos e intrácrinos, não é a mesma nos diferentes períodos da vida. A mulher nasce com um número determinado de folículos nos dois ovários, diminuindo gradualmente este número por atresia e/ou recrutamento para ovulação. Nas mais jovens, as taxas de atresia são baixas e as de crescimento folicular são altas. Há progressiva inversão nestas taxas ao longo dos anos reprodutivos ${ }^{26}$. Dos cerca de 6-7 milhões de folículos existentes em cada ovário na $20^{\mathrm{a}}$ semana de vida intra-uterina, o feto feminino nasce com 1 a 2 milhões; este período de vida intrauterino é marcado por uma perda rápida, na ordem de 50.000 folículos diariamente entre a $20^{\mathrm{a}}$ semana e o nascimento. Do nascimento à puberdade, a velocidade de consumo folicular é atenuada a 300-500 folículos/dia, permitindo que a mulher inicie sua vida reprodutiva com uma população de 300.000-500.000 folículos. Nos anos reprodutivos, a mulher perde cerca de 1.000 folículos a cada ciclo ou 30 folículos diariamente ${ }^{27}$.

Após os 35 anos, as alterações funcionais hipotálamo-hipofisárias, conseqüência da mudança temporal na organização dos sinais emitidos pelos marcapassos neurais, antecedem, em vários anos, a exaustão folicular e desencadeiam nova aceleração no consumo folicular. Numa mulher de 50 anos, com ciclos menstruais ainda regulares, cada ovário contém entre 2.500-4.000 folí$\operatorname{culos}^{28}$. É, então, a partir da segunda metade da quarta década que ocorre diminuição acentuada na população folicular. Esta diminuição é precedida pela elevação monotrópica de FSH e acompanhada clinicamente pelas primeiras alterações no intervalo dos ciclos menstruais ${ }^{29}$. Ainda, com o avançar da idade, parece haver diminuição da resposta ovariana a quantidades crescentes de gonadotrofinas, mas a elevação compensatória de FSH 
nem sempre é acompanhada de diminuição de estradiol ou inibina ${ }^{30}$

Modificações das gonadotrofinas na vida fetal, infância e puberdade

Moléculas de FSH e LH são secretadas pela hipófise fetal, de modo já pulsátil, a partir da $10^{a}$ semana de vida intrauterina. As concentrações de gonadotrofinas fetais permanecem estáveis e diminuem após a $25^{\mathrm{a}}$ semana de gravidez, provavelmente pela habilidade do eixo hipotálamo-hipofisário de responder negativamente ao estrogênio circulante nesta fase. Após o nascimento, os níveis de estradiol diminuem e permanecem em baixas concentrações até o início dos eventos puberais. A diminuição dos estrogênios no pós-parto é acompanhada de elevação nos níveis de LH e FSH, conseqüiente à atenuação do sistema de retrocontrole negativo. Entre o segundo e o quarto ano de vida e início da puberdade, há diminuição das gonadotrofinas circulantes por inibição intrínseca via $\mathrm{SNC}$ e neurônios $\mathrm{GnRH}$, via GABA-neurônios, eventos estes independentes dos esteróides ovarianos ${ }^{31}$. $\mathrm{Na}$ infância, o pulso de GnRH ocorre a intervalos de quatro horas $^{32}$. A partir de 7-8 anos, nas mulheres, hipotálamo e hipófise tornam-se menos sensíveis ao retrocontrole negativo do estradiol, resultando inicialmente em elevação noturna das concentrações de LH logo após a primeira hora de sono ${ }^{33}$. Nas mulheres, esta elevação está relacionada aos movimentos rápidos dos olhos e não ao ciclo noite-dia. Esta elevação, resultado de aumento na amplitude nos ainda irregulares pulsos de LH, é interrompida pela manhã. Seguindo na puberdade, os pulsos noturnos de LH tornam-se mais regulares, surge aumento na freqüência e amplitude destes pulsos e persistência dos mesmos durante o dia. Posteriormente, após amadurecimento funcional do eixo HHO e ovulação, há perda da secreção noturna diferenciada do LH, cerca de um ano após a menarca ${ }^{34}$. No final da infância, a hipófise torna-se mais sensível ao GnRH e o aumento de sua resposta à administração exógena experimental de $\mathrm{GnRH}$ antecipa o início da puberdade para os próximos 6-12 meses $^{35}$.

Modificações das gonadotrofinas na vida reprodutiva

Flutuações na amplitude e freqüência na liberação de $\mathrm{GnRH}$ combinadas com alterações na capacidade secretora do gonadotropo respondem pelas modificações nos níveis circulantes das gonadotrofinas durante os anos reprodutivos. Nesta fase, a função complexa e interdependente do eixo HHO resulta em níveis de gonadotrofinas variáveis de acordo com o período do dia, a fase do ciclo menstrual e anos decorridos após a menarca. Os níveis de FSH atingem níveis mais elevados no final da tarde e declinam entre 4 a $18 \%$ à noite, dependendo da fase do ciclo. Os níveis de $\mathrm{LH}$ diminuem em cerca de $15 \%$ durante a noite em todos as fases do ciclo menstrual ${ }^{36}$. Examinando o ciclo menstrual, tem-se pequena elevação de 10-30\% nos níveis de LH e FSH na fase lútea tardia, favorecendo o recrutamento do grupo de folículos antrais sensíveis às gonadotrofinas. A maior produção de estradiol com o recrutamento folicular assegura parada do fluxo menstrual. Com a seleção e crescimento do folículo dominante, há maior elevação de estradiol e diminuição gradual dos níveis de FSH, a partir da fase folicular média até a fase pré-ovulatória. Os níveis de LH permanecem estáveis ao longo da foliculogênese, sendo que menos de $1 \%$ dos receptores necessitam ser ocupados para que esta gonadotrofina exerça sua função ${ }^{37}$. A elevação nos níveis de estradiol na fase pré-ovulatória, por um período de pelo menos dois dias e em níveis acima de 200 pg/mL, promove a liberação em bolus das moléculas de $\mathrm{GnRH}$ na circulação porta hipofisária. Este efeito de retrocontrole hipotalâmico positivo coincide com a existência de gonadotropos repletos de moléculas de LH e FSH. Por meio da circulação porta hipofisária o gonadotropo é estimulado de modo agudo por uma onda de $\mathrm{GnRH}$, cerca de 24 horas após. O resultado é a elevação dos níveis de FSH de aproximadamente $5 \mathrm{UI} / \mathrm{mL}$ para $30-40 \mathrm{UI} / \mathrm{mL}$ e de $\mathrm{LH}$ de $5 \mathrm{mUI} / \mathrm{mL}$ para $40-80 \mathrm{mUI} / \mathrm{mL}^{38}$. Estes eventos, nomeados picos de LH e FSH, são transitórios e a elevação do estradiol na fase lútea não é acompanhada de elevação de LH e FSH devido ao período refratário existente após o pico ovulatório e a depleção dos receptores-GnRH no gonadotropo.

Em resposta ao aumento na secreção de progesterona, a freqüência dos pulsos de $\mathrm{LH}$, de aproximadamente um pulso por hora durante a fase lútea inicial, diminui para um pulso a cada 3-8 horas durante as fases lúteas média e tardia ${ }^{39}$. Importa notar que a regressão lútea não se deve a esta atenuação na pulsatilidade de LH, e sim à diminuição da resposta das células do corpo lúteo envelhecido às moléculas de LH. Durante toda a fase lútea, são baixos os níveis de LH, mas cerca de dois dias antes da menstruação há novo aumento na freqüência pulsátil desta gonadotrofina ${ }^{40}$. Até o final desta fase, devido à ação conjunta de estradiol, progesterona e inibina, os níveis de FSH mantêm-se abaixo do limiar necessário para estimular nova foliculogênese ${ }^{41}$. Os níveis médios de LH e FSH circulantes permanecem entre 3-10 mUI/mL ao longo de toda a fase lútea, havendo pequena elevação de FSH nos dias que antecedem a menstruação, por perda gradual do efeito inibitório da 

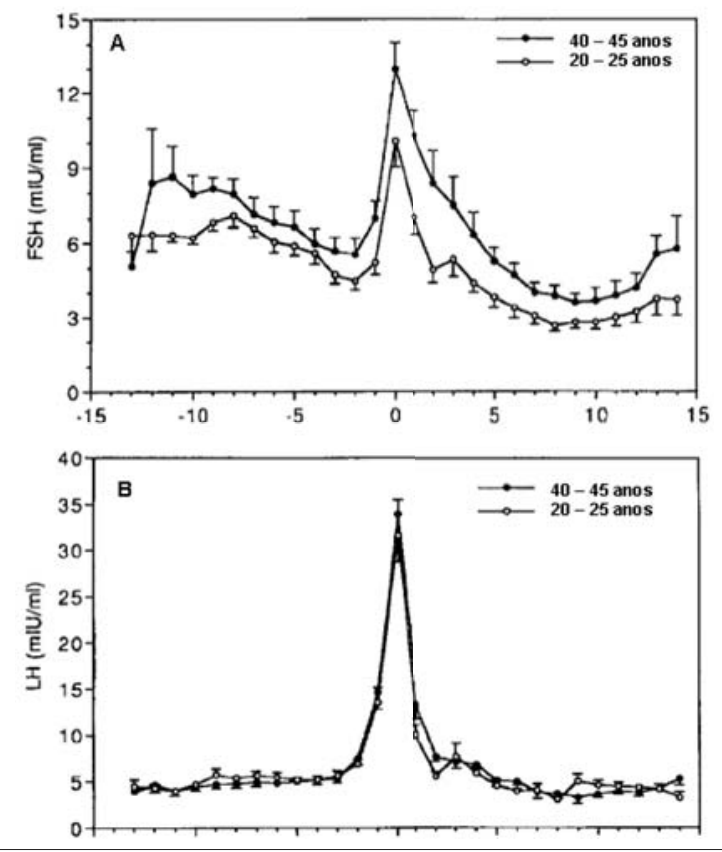

Figura 1- Média diária ( \pm EPM) dos níveis de FSH (Painel A) e LH (Painel B) durante o ciclo menstrual em mulheres entre 20 - 25 anos e 40 - 45 anos de idade. Adaptada da referência 46.

progesterona $^{42}$. Às modificações das gonadotrofinas com a fase do ciclo sobrepõem-se modificações decorrentes da própria idade (Figura 1, Painéis A e B).

Durante os anos reprodutivos, a média dos níveis basais de $\mathrm{LH}$, aferida na fase folicular precoce, ainda durante a menstruação, eleva-se lentamente e permanece abaixo de $10 \mathrm{mUI} / \mathrm{mL}$ até imediatamente antes da menopausa (Figura 2A). No entanto, alguns estudos registraram aumento mais marcante do LH com a idade, principalmente após 40-45 anos de idade ${ }^{43}$. Os níveis de FSH elevam-se lenta e gradualmente de um modo não linear em todas as idades, até a segunda metade da quarta década de vida ${ }^{44}$. Aos 37-38 anos, há elevação monotrópica rápida dos níveis de FSH (Figura 2B). Estes níveis refletem, indiretamente, a população folicular restante na córtex ovariana ${ }^{26}$. Nos últimos anos que antecedem a menopausa, a elevação de FSH é acompanhada de aumento variável na freqüência e amplitude dos pulsos de $\mathrm{LH}^{43,45}$. Discrepâncias nos valores basais de LH e FSH, observadas em alguns estudos, com variações entre 4-25 $\mathrm{mUI} / \mathrm{mL}$, podem ser resultado dos diferentes métodos empregados na quantificação destas gonadotrofinas e, certamente, devido à heterogeneidade da população incluída. Muitos estudos disponíveis não excluem mulheres com probabilidade maior de consumo folicular mais acelerado, possivelmente incluindo tabagistas, portadoras de galactosemia e aquelas submetidas

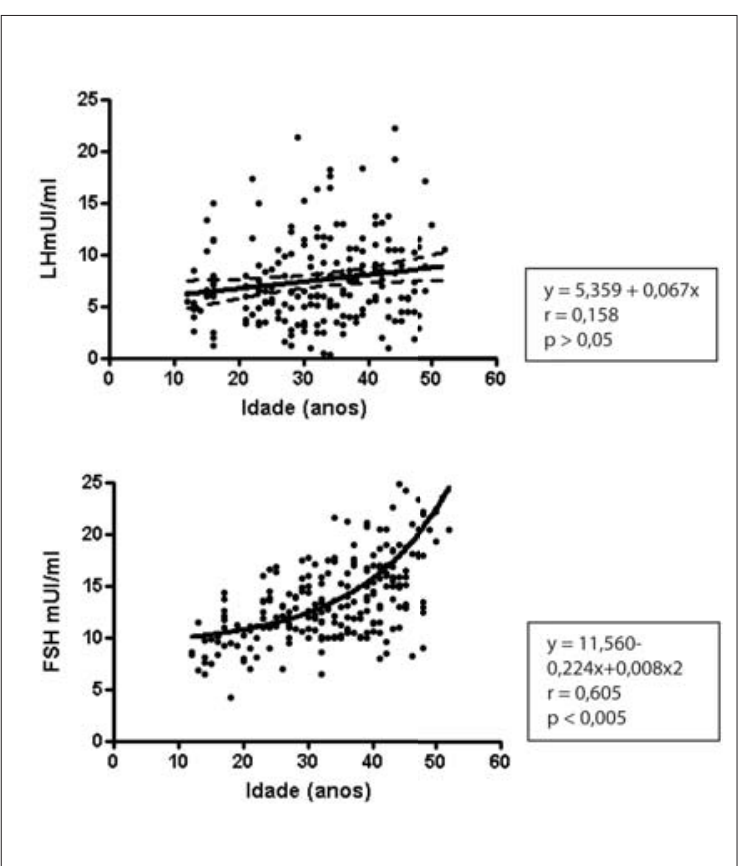

Figura 2 - Correlação entre idade cronológica e concentrações séricas de $\mathrm{LH}$ (Painel A) e FSH (Painel B) na fase folicular precoce. Adaptada, pela retirada de outliers (valores inconsistentes) e redesenhada da referência 44.

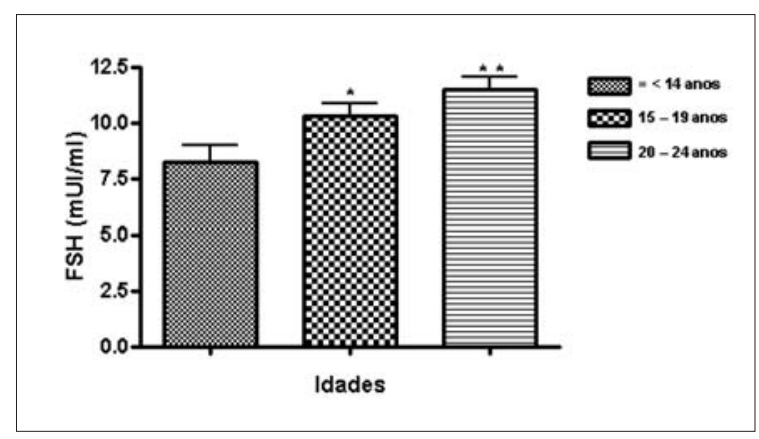

Figura 3 - Comparação entre os níveis basais de FSH em mulheres com até 14 anos de idade, entre 15 e 19 anos e entre 20 e 24 anos. Análise de variância e teste de Dunnett dos dados da tabela 1, * $p=0,057$, * * $p<0,01$, referência 3 .

a cirurgias ginecológico-ovarianas, variáveis com possível repercussão sobre a reserva ovariana ${ }^{3,44}$. Após exclusão das mulheres com risco de antecipação da falha ovariana, os níveis de FSH deveriam ser estabelecidos de modo pontual em mulheres saudáveis nas diferentes faixas etárias. Faltam estudos neste sentido.

Evidências recentes mostram que os níveis de FSH elevam-se precocemente mesmo em mulheres com menos de 20 anos de idade (Figura 3). Em números absolutos, nas mulheres com ciclos ainda regulares, os níveis de FSH crescem entre 2-3 vezes quando se comparam 
mulheres abaixo de 15 anos com aquelas com mais de 45 (em valores aproximados, de $8,0 \mathrm{mUI} / \mathrm{mL}$ para $22,0 \mathrm{mUI} / \mathrm{mL})^{44}$. A marcante e brusca elevação após os 37 anos prediz a ocorrência de menopausa com 7-10 anos de antecedência. Apesar desta elevação de FSH no período de transição para a menopausa, os níveis de estradiol podem estar normais ou elevados. Especula-se a existência de pelo menos dois grupos de mulheres com resposta distinta: um com elevação de ambos FSH e estradiol e outro com elevação de FSH e níveis estáveis ou diminuídos deste esteróide. A proporção de mulheres com níveis de $\mathrm{FSH}>15 \mathrm{mUI} / \mathrm{mL}$ é de $15 \%$ antes dos 35 anos, elevando-se para $56 \%$ nas mulheres com mais de 35 anos $^{3}$. Incorporando a nova proposta de estagiar o envelhecimento reprodutivo, já no estágio 3, entre 2530 anos, há elevação do FSH em dois desvios padrões acima dos níveis das mulheres na segunda década de vida (Figura 3). Esta elevação precoce tem sido aceita como o primeiro sinal de envelhecimento reprodutivo ${ }^{32} \mathrm{e}$ atribuída à diminuição da inibina ${ }^{5}$. Devido à sua menor sensibilidade aos mecanismos de retrocontrole negativo exercido pelos esteróides e peptídeos ovarianos, os níveis de $\mathrm{LH}$ permanecem abaixo de $10 \mathrm{mUI} / \mathrm{mL}$ até meados da quinta década de vida, elevando-se cerca de cinco a dez anos após a elevação seletiva de $\mathrm{FSH}^{44-46}$, geralmente a partir dos 45 anos de idade, quando já existem modificações marcantes no padrão menstrual ${ }^{7,43,47}$.

\section{Conclusão}

Os mecanismos fisiológicos responsáveis pelas modificações das gonadotrofinas antes, durante e após os anos reprodutivos envolvem uma rede de neurônios do tronco cerebral e hipotálamo que asseguram liberação pulsátil de $\mathrm{GnRH}$, na interdependência orquestrada de neurotransmissores e esteróides ovarianos. Alterações na freqüência e duração dos pulsos de $\mathrm{GnRH}$ e produção de quantidades variáveis de estradiol e progesterona explicam as oscilações de LH e FSH ao longo da vida. Por outro lado, moduladores locais nas células foliculares da granulosa e teca determinam a velocidade de consumo folicular. Os mecanismos que operam na vida intrauterina, infância e anos reprodutivos são temporalmente diferentes. A própria idade cronológica parece ter participação relevante apenas nas modificações dos níveis de FSH. Durante o menacme, as concentrações basais de LH permanecem quase constantes, sofrendo modificações ao longo do ciclo menstrual. As concentrações basais de FSH, por outro lado, elevam-se gradualmente à medida que diminuiu o número de folículos ovarianos. Após os 37-38 anos de idade, há elevação monotrópica nas concentrações de FSH e aceleração da velocidade no consumo folicular. Este evento ainda não totalmente compreendido parece sincronizado com o envelhecimento funcional hipotalâmico. Há necessidade de maiores estudos acerca das modificações na sensibilidade dos neurônios-GnRH e perda ou atenuação do mecanismo de retrocontrole positivo do estradiol na quarta década de vida. Do mesmo modo, há ainda necessidade de maior compreensão sobre a secreção preferencial de moléculas de FSH pelo gonadotropo após os 37 anos de idade e os mecanismos íntimos responsáveis pela ocorrência de nova aceleração do consumo folicular nesta fase. Na prática clínica, há necessidade de se estabelecerem valores de referência para o FSH nas diferentes idades, tanto em mulheres saudáveis como nas portadoras de risco de consumo folicular mais acelerado.

\section{Referências}

1. Davis PJ. Ageing and endocrine function. Clin Endocrinol Metab. 1979; 8(3):603-19. Review.

2. Burger HG, Dudley EC, Robertson DM, Dennerstein L. Hormonal changes in the menopause transition. Recent Prog Horm Res. 2002; 57(1):257-75.

3. Medeiros SF, Assi PE, Sékula VG, Carmona F, Yamamoto MMW. Dinâmica dos níveis do hormônio folículo estimulante na fase folicular precoce durante a vida reprodutiva. Reprod Clim. 2002; $17(1): 34-8$.

4. Wise PM, Scarbrough K, Lloyd J, Cai A, Harney J, Chiu S, et al. Neuroendocrine concomitants of reproductive aging. Exp Gerontol. 1994; 29(3-4):275-83

5. Klein NA, Illingworth PJ, Groome NP, McNeilly AS, Battaglia DE, Soules MR. Decreased inhibin B secretion is associated with the monotropic $\mathrm{FSH}$ rise in older, ovulatory women: study of serum and follicular fluids levels of dimeric inhibin A and B in spontaneous menstrual cycles. J Clin Endocrinol Metab. 1996; 81 (7):2742-5.

6. Azcoitia I, DonCarlos LL, Garcia-Segura LM. Are gonadal steroid hormones involved in disorders of brain aging? Aging Cell. 2003; 2(1):31-7.

7. Dudas B, Merchenthaler I. Three-dimensional representation of the neurotransmitter systems of the human hypothalamus: inputs of the gonadotrophin hormone-releasing hormone neuronal system. J Neuroendocrinol. 2006; 18(2):79-95.

8. Nunemaker CS, DeFazio RA, Geusz ME, Herzog ED, Pitts GR, Moenter SM. Long-term recordings networks of immortalized $\mathrm{GnRH}$ neurons reveal episodic patterns of electrical activity. J Neurophysiol. 2001; 86(1):86-93.

9. Leng $G$, Brown $D$. The origins and significance of pulsatility in hormone secretion from the pituitary. J Neuroendocrinol. 1997; 9(7):493-513. 
10. Halasz B, Kiss J, Molnar J. Regulation of the gonadotropin-releasing hormone $(\mathrm{GnRH})$ neuronal system: morphological aspects. J Steroid Biochem. 1989; 33(4B):663-8.

11. Veldhuis JD. The hypothalamic pulse generator: the reproductive core. Clin Obstet Gynecol. 1990; 33(3):538-50

12. McCartney CR, Gingrich MB, Hu Y, Evans WS, Marshall JC. Hypothalamic regulation of cyclic ovulation: evidence that the increase in gonadotropin-releasing hormone pulse frequency during the follicular phase reflects the gradual loss of the restraining effects of progesterone. J Clin Endocrinol Metab. 2002; 87(5):2194-200.

13. Bilezikjian $L M$, Blount $A L$, Leal $A M$, Donaldson CJ, Fischer WH, Vale WW. Autocrine/paracrine regulation of pituitary function by activin, inhibin and follistatin. Mol Cell Endocrinol. 2004; 225(1-2):29-36.

14. Yin W, Gore AC. Neuroendocrine control of reproductive aging: roles of $\mathrm{GnRH}$ neurons. Reproduction. 2006; 131 (3):403-14.

15. Miller MM, Bennett HP, Billar RB, Franklin KB, Joshi D. Estrogen, the ovary, and neutotransmitters: factors associated with aging. Exp Gerontol. 1998; 33(7-8):729-57.

16. Quadri SK, Kledzik GS, Meites J. Reinitiation of estrous cycles in old constant-estrous rats by central-acting drugs. Neuroendocrinology. $1973 ; 11(4): 248-55$.

17. Van der Beek EM, Wiegant VM, van der Donk HA, van den Hurk $\mathrm{R}$, Buijs RM. Lesions of the suprachiasmatic nucleus indicate the presence of a direct vasoactive intestinal polypeptide-containing projection to gonadotrophin-releasing hormone neurons in the female rat. J Neuroendocrinol. 1993; 5(2):137-44

18. Brann DW, Mahesh VB. The aging reproductive neuroendocrine axis. Steroids. 2005; 70(4):273-83.

19. Rossmanith WG. Gonadotropion secretion during aging in women: review article. Exp Gerontol. 1995; 30(3-4):369-81.

20. Matt DW, Kauma SW, Pincus SM, Veldhuis JD, Evans WS. Characteristics of luteinizing hormone secretion in younger versus older premenopausal women. Am J Obstet Gynecol. 1998; 178(3):504-10.

21. Rehman HU, Masson EA. Neuroendocrinology of female aging Gend Med. 2005; 2(1):41-56. Review.

22. Kaiser UB, Sabbagh E, Katzenellenbogen RA, Conn PM, Chin WW. A mechanism for the differential regulation of gonadotropin subunit gene expression by gonadotropin-releasing hormone. Proc Natl Acad Sci. 1995; 92(26): 12280-4.

23. Chen $\mathrm{H}$. Gene expression by the anterior pituitary gland: effects of age and caloric restriction. Mol Cell Endocrinol. 2004; 222(1-2):21-31.

24. Sharpless JL, Supko JG, Martin KA, Hall JE. Disappearance of endogenous luteinizing hormone is prolonged in postmenopausal women. J Clin Endocrinol Metab. 1999; 84(2):688-94.

25. Hall JE, Lavoie HB, Marsh EE, Martin KA. Decrease in gonadotropinreleasing hormone ( $\mathrm{GnRH}$ ) pulse frequency with aging in postmenopausal women. J Clin Endocrinol Metab. 2000; 85(5):1794-800.

26. Faddy M. Follicle dynamics during ovarian ageing. Mol Cell Endocrinol. 2000; 163(1-2):43-8.

27. Medeiros SF, Yamamoto MM. Mecanismos do consumo folicular ovariano. Reprod Clim. 1998; 13(1):18-27.

28. Gougeon A, Ecochard R, Thalabard JC. Age-related changes of the population of human ovarian follicles: increase in the disappearance rate of non-growing and early-growing follicles in aging women. Biol Reprod. 1994; 50(3):653-63.

29. Faddy M, Gosden RG, Gougeon A, Richardson SJ, Nelson JF. Accelerated disappearance of ovarian follicles in mid-life: implications for forecasting menopause. Hum Reprod. 1992; 7(10): 1342-6
30. Jacobs SL, Metzger DA, Dodson WC, Haney AF. Effect of age on response to human menopausal gonadotropin stimulation. J Clin Endocrinol Metab. 1990; $71(6): 1525-30$

31. Delemarre-van de Waal HA. Regulation of puberty. Best Pract Res Clin Endocrinol Metab. 2002; 16(1):1-12. Review.

32. Park SJ, Goldsmith LT, Weiss G. Age-related changes in the regulation of luteinizing hormone secretion by estrogen in women. Exp Biol Med (Maywood). 2002; 227(7):455-64.

33. Apter D, Butzow TL, Laughlin GA, Yen SS. Gonadotropin-releasing hormone pulse generator activity during pubertal transition in girls: pulsatile and diurnal patterns of circulating gonadotropins. J Clin Endocrinol Metab. 1993; 76(4):940-9.

34. Terasawa E, Fernandez DL. Neurobiological mechanisms of the onset of puberty in primates. Endocr Rev. 2001; 22(1):111-51.

35. Styne DM. Physiology of puberty. Horm Res. 1994; 41 Suppl 2 3-6. Review.

36. Mortola JF, Laughlin GA, Yen SS. A circadian rhythm of serum follicle-stimulating hormone in women. J Clin Endocrinol Metab. $1992 ; 75(3): 861-4$.

37. Chappel SC, Howles C. Reevaluation of the roles of luteinizing hormone and follicle-stimulating hormone in the ovulatory process. Hum Reprod. 1991; 6(9):1206-12

38. Shoham Z, Schacter M, Loumaye E, Weissman A, MacNamee M, Insler $\mathrm{V}$. The luteinizing hormone surge-the final stage in ovulation induction: modern aspects of ovulation triggering. Fertil Steril. $1995 ; 64(2): 237-51$.

39. Wuttke W, Theiling K, Hinney B, Pitzel L. Regulation of steroid production and its function within the corpus luteum. Steroids. 1998; 63(5-6):299-305

40. Zeleznik AJ. Modifications in gonadotropin signaling: a key to understanding cyclic ovarian function. J Soc Gynecol Investig. 2001; 8(1 Suppl Proceedings):S24-5. Review.

41. Stouffer RL. Progesterone as a mediator of gonadotrophin action in the corpus luteum beyond steroidogenesis. Hum Reprod Update. 2003; 9(2):99-117.

42. Rossmanith WG, Laughlin GA, Mortola JF, Johnson ML, Veldhuis JD, Yen SS. Pulsatile consecretion of estradiol and progesterone by the midluteal phase corpus luteum: temporal link to luteinizing hormone pulses. J Clin Endocrinol Metab. 1990; 70(4):990-5.

43. Ferrell RJ, O'Connor KA, Holman DJ, Brindle E, Miller RC, Rodriguez $G$, et al. Monitoring reproductive aging in a 5 -year prospective study: aggregate and individual changes in luteinizing hormone and follicle-stimulating hormone with age. Menopause. 2006 Oct 2; [Epub ahead of print]

44. de Medeiros SF, Assi PE, de Medeiros MM. Gonadotrophin dynamics during reproductive life. Int J Gynaecol Obstet. 2004 87(1):24-8.

45. Reame NE, Kelch RP, Beitins IZ, Yu MY, Zawacki CM, Padmanabhan V. Age effects on follicle-stimulating hormone and pulsatile luteinizing hormone secretion across the menstrual cycle of premenopausal women. J Clin Endocrinol Metab. 1996; $81(4): 1512-8$

46. Klein NA, Battaglia DE, Clifton DK, Bremner WJ, Soules MR. The gonadotropin secretion pattern in normal women of advanced reproductive age in relation to the monotropic FSH rise. J Soc Gynecol Investig. 1996; 3(1):27-32.

47. Ahmed Ebbiary NA, Lenton EA, Cooke ID. Hypothalamic-pituitary ageing: progressive increase in $\mathrm{FSH}$ and $\mathrm{LH}$ concentrations throughout the reproductive life in regularly menstruating women. Clin Endocrinol (Oxf). 1994; 41 (2):199-206. 